\title{
Evaluation of Tooth Surface Irradiated With Erbium: Yttrium Aluminum Garnet and Carbon Dioxide Lasers by Atomic Force Microscopy
}

\author{
Sogol Saberi ${ }^{1}$, Sooreh Seyed Jabbari Doshanlo², Hossein Bagheri ${ }^{3}$, Susan Mir Mohammad Rezaei ${ }^{4}$, \\ Sima Shahabi ${ }^{1,5^{*}}$ \\ 'Laser Research Center of Dentistry, Dentistry Research Institute, Tehran University of Medical Sciences, Tehran, Iran \\ ${ }^{2}$ Periodontics Department, School of Dentistry, Shahid Beheshti University of Medical Science, Tehran, Iran \\ ${ }^{3}$ Department of Dental Material, Dental Material Research Center, Faculty of Dentistry, Mashhad University of Medical \\ Science, Mashhad, Iran \\ ${ }^{4}$ Department of Prosthodontics, Tehran University of Medical Sciences, Tehran, Iran \\ ${ }^{5}$ Dental Biomaterials Department, School of Dentistry, Tehran University of Medical Sciences, Tehran, Iran
}

\section{*Correspondence to Sima Shahabi, Dental Biomaterials Department, School of Dentistry, Tehran University of Medical Sciences, Tehran, Iran. Tel: +989124264904; Email: shahabis@tums.ac.ir}

Published online 28 July 2018

\begin{abstract}
Introduction: Laser irradiation of dentin surface can affect its surface topography and roughness. Atomic force microscopy (AFM) is among the most efficient tools for determination of surface topography of natural biomolecules in nano-scales. Surface roughness affects plaque retention especially in the cervical region. This study aimed to assess and compare the obstruction of dentinal tubules and dentin surface roughness after irradiation of erbium: yttrium aluminum garnet (Er:YAG ) and $\mathrm{CO} 2$ lasers for treatment of dentin hypersensitivity $(\mathrm{DH})$.

Methods: Five disc-shaped samples measuring $3 \mathrm{~mm}$ in thickness were fabricated by horizontal sectioning of the cervical area of five extracted human molars using a low speed saw. Each disc was divided into 3 segments by a bur under water coolant. The three segments of each disc were placed on a glass slide. First segment: No intervention (control group). Second segment: Er:YAG laser irradiation ( $2940 \mathrm{~nm}, 50 \mathrm{~mJ}, 10 \mathrm{~Hz}$ and $0.5 \mathrm{~W}, 30$ seconds) along with water coolant. Third segment: CO2 laser irradiation ( $10600 \mathrm{~nm}, 80 \mathrm{~Hz}, 0.3 \mathrm{~W}, 30$ seconds) along with water coolant. After that, the surfaces underwent non-contact AFM. The diameters of dentinal tubules as well as surface roughness were then measured and statistically analyzed using repeated measures ANOVA.

Results: The surface roughness parameters $(\mathrm{Ra}, \mathrm{Rq})$ showed increased roughness after laser irradiation and this increase in roughness after Er:YAG laser application was significant compared to the control group $(P=0.048)$. However, CO2 laser caused no significant change in surface roughness. Also, after Er:YAG laser application, fewer open dentinal tubules were observed and the remaining open tubules had a smaller diameter.

Conclusion: Based on the results, Er:YAG laser irradiation obstructs the dentinal tubules and increases the dentin surface roughness. This increase in surface roughness can cause microbial plaque retention and increase the risk of caries and periodontal disease. Application of CO2 laser (compared to Er:YAG) lead to slight but clinically significant obstruction of dentinal tubules and surface roughness

Keywords: Dental tubule; Dentin hypersensitivity; Laser; Atomic force microscopy.
\end{abstract}

\section{Introduction}

Dentin hypersensitivity (DH) is defined as pain due to exposure of dentin. This pain is brief and sharp and produced in response to chemical, osmotic, and contact stimuli. This pain cannot be attributed to any pathological condition. ${ }^{1}$ The global prevalence of $\mathrm{DH}$ has been reported to be $10 \%$ to $30 \%$ depending on the population and study design. ${ }^{2}$ It most commonly involves patients in the age range of 20 to 30 years. ${ }^{3}$ $\mathrm{DH}$ occurs because of loss of protective coat of dentin, and its exposure to the oral environment. ${ }^{4}$ It often takes place due to abrasion, attrition, erosion, abfraction, gingival recession, and incorrect tooth brushing habits. ${ }^{5}$ The mechanism of $\mathrm{DH}$ has been the subject of many recent investigations. Several mechanisms have been proposed explaining $\mathrm{DH}$, but three mechanisms are more commonly accepted. ${ }^{6}$ First mechanism is direct innervation theory: Free nerve endings are widely spread in dentin, and react upon direct stimulation of dentin. ${ }^{7}$ Second one is Odontoblastic Receptor theory (OR):

Please cite this article as follows: Saberi S, Seyed Jabbari Doshanlo S, Bagheri H, Mir Mohammad Rezaei S, Shahabi S. Evaluation of tooth surface irradiated with erbium: yttrium aluminum garnet and carbon dioxide lasers by atomic force microscopy. I Lasers Med Sci. 2018;9(3):188-193. doi:10.15171/jlms.2018.34. 
Odontoblasts may be traumatized because of thermal, mechanical, chemical or osmotic stimulation of dentin. Based on this theory, odontoblasts and their processes can serve as receptors. Upon stimulation of their cell membrane, they send an electric or chemical pain signal. ${ }^{7}$ The last mechanism is Hydrodynamic theory: Evidence shows that dentinal fluid flow is the main cause of pain. The pain stimulators like heat and cold result in the flow of dentinal fluid. ${ }^{8}$ Researchers found a positive correlation between the flow rate of dentinal fluid and the degree of excitation of dentinal nerves. They noticed that fluid outflow causes a more severe response than inflow of the fluid. ${ }^{8}$ To make an accurate diagnosis, the causative agents and the modifying factors must be well assessed. Accurate diagnosis includes a complete history taking and clinical and radiographic examinations. ${ }^{9}$ Several methods have been used for treatment of DH. Most of these methods are suitable for treatment of hypersensitivity of the cervical region of the teeth. ${ }^{10}$ Clinicians use different materials and techniques for treatment of $\mathrm{DH}$ including special desensitizing tooth pastes, laser irradiation, dentin adhesives, antibacterial agents, resin materials, and fluoride-containing mouthwashes. ${ }^{11}$ Application of laser for treatment of tooth hypersensitivity was first reported by Matsumoto et al in 1985. They used Nd:YAG laser for this purpose. ${ }^{12}$ Advances in laser technology have increased its application in dentistry and for treatment of tooth hypersensitivity. ${ }^{13}$ Laser decreases tooth hypersensitivity via two mechanisms: the first mechanisms is, direct effect of laser on electrical activity of nerve fibers present in dental pulp, and the second mechanism is, obstruction of dentinal tubules via the melting phenomenon. ${ }^{14}$ Two types of lasers may be used for treatment of tooth hypersensitivity, low-level lasers including $\mathrm{He}-\mathrm{Ne}$, diode and GaAlAs and intermediatelevel lasers including $\mathrm{Nd}$ YAG, $\mathrm{CO}_{2}$, Er:YAG and Er,Cr:YSGG.${ }^{14} \mathrm{CO}_{2}$ laser was first used for treatment of tooth hypersensitivity by Moritz et al, ${ }^{15}$ in 1996 in 5 W power for 5 seconds in 6 sessions. The mechanism of this effect was through obstruction and narrowing of dentinal tubules. ${ }^{16}$ The sealing (obstruction) depth of dentinal tubules by $2 \mathrm{~W} \mathrm{CO}$ laser and 1 second time was found to be approximately 2-8 microns. ${ }^{14}$ Er:YAG laser at $2940 \mathrm{~nm}$ wavelength was used by Shwartz et al, ${ }^{17}$ in 2002 . This laser is highly absorbed by water and results in evaporation of the dentinal fluid and the smear layer and consequently leads to obstruction of dentinal tubules. Another possible mechanism may be related to the melting phenomenon by use of this laser. ${ }^{14}$ Laser application for treatment of $\mathrm{DH}$ has been associated with controversial results. Some researchers attributed its effects to the placebo effect, ${ }^{18}$ while some others have shown equal or higher efficacy of laser alone or in combination with chemical agents such as GLUMA, and sodium fluoride than that of chemical methods for treatment of DH. ${ }^{19,20}$ Atomic force microscopy (AFM) is among the most efficient tools for determination of surface topography of natural biomolecules in nano-scales. In scanning AFM, the surface of specimen is probed by a sharp needle with 2 $\mu$ length; its tip diameter is often $10 \mathrm{~nm}$. The needle is located at the free end of a cantilever with 100-400 $\mu$ length. The loads between the needle and specimen surface bring about bending or deviation of the cantilever. An indicator measures the deviation of the cantilever when the needle scans the specimen surface. Measurement of the cantilever deviation enables the computer to produce an image of the surface topography. Scanning AFM can be used to study the conductive, semi-conductive, and non-conductor materials. Different forces participate in deviations of scanning AFM such as atomic or van der Waals forces. Most currently used AFMs have laser beam refraction system. ${ }^{21}$

Laser irradiation may change surface topography and surface roughness that increasing dentin surface roughness. ${ }^{22}$ There is a high positive correlation between surface roughness and bacterial adhesion. ${ }^{23}$ Also, increased plaque retention compromises periodontal health. ${ }^{24}$ Not many studies have assessed the effect of laser irradiation or the irradiation settings for treatment of $\mathrm{DH} .{ }^{25}$ Thus, there was an obvious need to assess the effect of laser irradiation on changes in dentin surface and surface roughness.

This study sought to assess and compare the obstruction of dentinal tubules and dentin surface roughness after Er:YAG and $\mathrm{CO}_{2}$ laser irradiation for treatment of $\mathrm{DH}$.

\section{Methods}

\section{Specimen Preparation}

Five disc-shaped samples were fabricated of the cervical region of five extracted human molars by horizontal sectioning using a low speed saw (IsoMet ${ }^{\circledR}$ Low Speed Saw, Buehler). The teeth were selected among mature third molars, which were preferably impacted and had no caries. The teeth were immersed in $0.5 \%$ chloramine $\mathrm{T}$ solution for one week for disinfection. After that the pulp tissue at the center of samples was removed, and all 5 discs were divided into 3 segments using a bur under water coolant and cementum was removed by bur under water coolant to expose the underlying dentin.

To eliminate the smear layer, the exposed dentin surface was subjected to $14 \%$ EDTA for 60 seconds and was then rinsed with sodium hypochlorite. Tooth samples were separately glued on glass slides. The three segments of each disc underwent the following interventions with laser

First segment: No intervention (control group)

Second segment: Er:YAG laser (2940D plus, Deka, Italy) irradiation with the irradiation setting of $50 \mathrm{~mJ}, 10 \mathrm{~Hz}$ and $0.5 \mathrm{~W}$ for 30 seconds with scanning motion and 4 $\mathrm{mm}$ distance with tip less handpiece.

Third segment: $\mathrm{CO}_{2}$ laser (US-20D, DEKA, Italy) irradiation with the irradiation settings of $10600 \mathrm{~nm}, 80$ 
$\mathrm{Hz}$ and $0.3 \mathrm{~W}$ for 30 seconds with scanning motion and $15 \mathrm{~mm}$ distance with tip less handpiece.

The samples were then subjected to non-contact AFM (JPK NanoWizard ${ }^{\circledR}$, Germany).

(a) Assessment of the diameter of dentinal tubules: images $(10 \times 10 \mathrm{~nm})$ were captured and descriptively analyzed.

(b) Measurement of surface roughness: The surface roughness ( $\mathrm{Ra}$ and $\mathrm{Rq}$ ) was determined in selected areas. The $\mathrm{Ra}$ and $\mathrm{Rq}$ values were measured in five points in each segment with equal distances from each other (1 $\mu \mathrm{m})$ and the mean of $\mathrm{Ra}$ and $\mathrm{Rq}$ was calculated. The $P$ values of surface roughness parameters were subjected to pair wise comparisons by Sidak test.

\section{Results}

Assessment of changes in surface roughness parameters and dentinal tubule diameters of the samples after laser irradiation:

Laser irradiation of dentin surface affected the surface topography and surface roughness of dentin. Evaluation of changes in surface roughness parameters ( $\mathrm{Ra}$ and $\mathrm{Rq}$ ) after laser irradiation revealed that surface roughness increased following laser irradiation.

- $\mathrm{Ra}$ parameter (arithmetic mean value of surface roughness), indicates the average roughness and is defined as the arithmetic mean deviation of the surface valleys and peaks from the center line in the measuring length.

- $\quad \mathrm{Rq}$ parameter (dentin mean square roughness), the
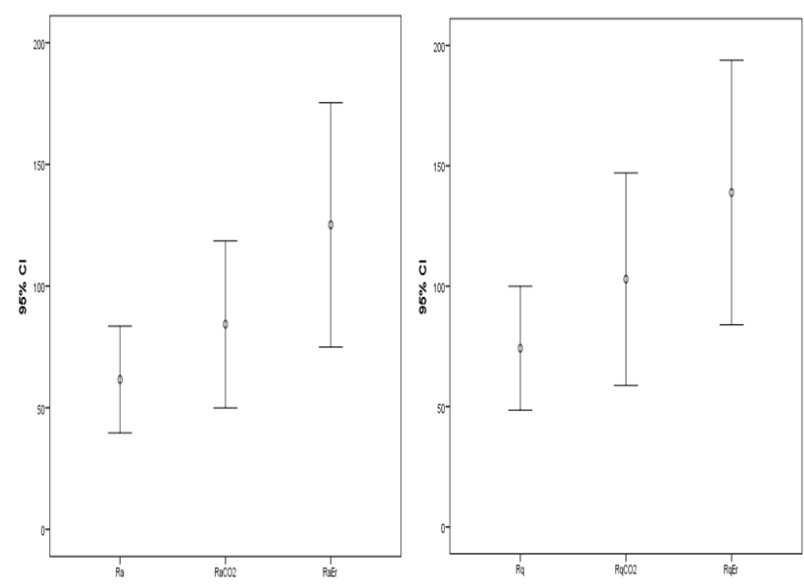

Figure 1. The Mean and $95 \% \mathrm{Cl}$ of 2 Surface Roughness Parameters After Laser Irradiation. average of the measured height deviations taken within the evaluation length or area and measured from the mean linear surface. $\mathrm{Rq}$ is the rms parameter corresponding to Ra.

The confidence interval and the mean value of the surface roughness parameters ( $\mathrm{Ra}$ and $\mathrm{Rq}$ ) increased in the intervention groups compared to the control group (Figure 1) and this increase in Er:YAG laser group was statistically significant compared to the control group.

Table 1 shows the mean and standard deviation (SD) of surface roughness parameters. As can be seen from the Table 1, the surface roughness parameters in both laser groups increased compared to the control group but this increase only in Er:YAG laser group was statistically significant.

Table 2 shows the $P$ values for the pair wise comparison of surface roughness parameters using the Sidak test. As seen from the Table 2, the increase in both surface roughness parameters $(\mathrm{Ra}, \mathrm{Rq})$ in Er:YAG laser group was significant compared to the control group $(P=0.04)$.

Figure 2 shows the AFM micrographs of the samples. As seen, the 2 intervention groups had fewer open dentinal tubules. The diameter of the remaining open tubules in both groups was smaller than that in the control group.

\section{Discussion}

In previous studies different chemical and mechanical methods, laser irradiation and ultrasound have been introduced for smear layer removal. ${ }^{26}$ Use of ethylenediaminetetraacetic acid (EDTA) for more than 20 minutes can result in over-demineralization and breakage of dentinal tubules. The efficacy of EDTA for smear layer removal is attributed to its ability in dissolving the inorganic components of the smear layer by elimination of calcium ions; for elimination of residual organic compounds, researchers have recommended its application in conjunction with sodium hypochlorite. ${ }^{22}$ Yamada et al, in 1983 noticed that 17\% EDTA along with sodium hypochlorite was more effective in smear layer removal than $25 \%$ citric acid combined with sodium hypochlorite. ${ }^{27}$ In our study, this material was applied for smear layer removal and exposure of dentinal tubules. Several methods have been recommended for $\mathrm{DH}$ treatment. Depending on the type and irradiation parameters of laser, it can have $5.2 \%$ to $100 \%$ efficacy. ${ }^{23}$ The mechanism of laser for treatment of $\mathrm{DH}$ has not been

Table 1. The Mean and Standard Deviation of Changes in Surface Roughness Parameters in the 2 Laser Groups After Laser Irradiation

\begin{tabular}{|c|c|c|c|c|c|}
\hline Surface Roughness Parameters & Mean & Median & Minimum & Maximum & Standard Deviation \\
\hline $\mathrm{Ra}$ & 61.60 & 50.62 & 46.19 & 82.75 & 17.67 \\
\hline $\mathrm{RaCO}_{2}$ & 84.28 & 84.24 & 49.81 & 125.55 & 27.65 \\
\hline RaEr & 125.16 & 102.95 & 92.03 & 189.66 & 40.45 \\
\hline $\mathrm{Rq}$ & 74.23 & 61.66 & 56.55 & 100.30 & 20.71 \\
\hline $\mathrm{RqCO}_{2}$ & 102.93 & 103.21 & 59.06 & 156.85 & 35.55 \\
\hline $\mathrm{RqEr}$ & 138.92 & 121.82 & 113.02 & 217.80 & 44.27 \\
\hline
\end{tabular}


Table 2. $P$ Values for Pair Wise Comparisons of Surface Roughness Parameters

\begin{tabular}{lc}
\hline Surface Roughness Parameters & P Value \\
\hline Ra versus Ra- $\mathrm{CO}_{2}$ & 0.24 \\
\hline Ra versus Ra-Er:YAG & 0.048 \\
Ra-Er:YAG versus Ra-CO & 0.18 \\
Rq versus Rq-CO & 0.22 \\
Rq versus Rq-Er:YAG & 0.04 \\
Rq-Er:YAG versus Rq-CO & 0.10 \\
\hline
\end{tabular}

well elucidated. However, Pashley stated that decreased $\mathrm{DH}$ might be related to changes in dentinal fluid flow or alterations in activity of nerve fibers. ${ }^{28}$ McCarthy et al showed that $\mathrm{DH}$ was due to the physical obstruction of dentinal tubules. ${ }^{29}$

In 2006, Crespi et al demonstrated using Er:YAG laser led to rougher surface morphology compared to the control group with no surface treatment. ${ }^{30}$

In 2011, Botta et al evaluated the effect of Er:Cr:YSGG laser with $12.5 \mathrm{~mJ}, 20 \mathrm{~Hz}$ frequency and $2.78 \mu \mathrm{m}$ wavelength parameters on micro-topography of dentin using AFM. They observed that after laser irradiation and comparison with the no-intervention control group, $\mathrm{Ra}$ significantly increased. ${ }^{31}$

In 2002, Schwarz et al revealed that Er:YAG laser irradiation with $3 \mathrm{~Hz}$ frequency and $80 \mathrm{~mJ}$ energy parameters had an efficacy below the required threshold for dentin ablation. Decreased flow of fluid in dentinal tubules based on the hydrodynamic theory is directly related to decreased $\mathrm{DH}$. The effect of laser on tissue depends on its energy intensity, radiation time and use of water coolant. ${ }^{32}$ However, in our study, higher energy and frequency parameters were used. Irrespective of other factors, surface roughness created in the dentin surface can be due to laser ablation.

Surface roughness created by Er:YAG laser irradiation $(100 \mathrm{~mJ}, 3 \mathrm{~Hz}, 60$ seconds) was the result of ablation of dentin surface in Birang et al study in 2007. In addition to causing surface roughness, laser seals the dentinal tubules and prevents the penetration of bacteria into dentinal tubules and thus reduces $\mathrm{DH} .{ }^{33}$

Er:YAG laser is an effective tool for elimination of smear layer from the root canal walls. Deposition of insoluble salts into exposed dentinal tubules results in obstruction of dentinal tubules and decreases DH. The efficacy of medical and dental applications of this type of laser is due to its absorption by water molecules and high thermomechanical ablation. The absorption of Er:YAG laser by water molecules is 15 times the rate for $\mathrm{CO}_{2}$ laser.

Soares et al study on third molars using scanning electron microscopy (SEM) showed that Er:YAG laser with an energy intensity above $180 \mathrm{~mJ}$ partially occluded the dentinal tubules and was not suitable for diffusion of materials. It more commonly affected the carbonate, phosphate and organic components of dentin. ${ }^{34}$ The results of the afore-mentioned study showed increased dentin surface roughness following the application of Er:YAG laser, which is in agreement with our findings. This is important considering the increased plaque retention on laser irradiated surfaces for the purpose of treatment of $\mathrm{DH}$. Irradiation of $\mathrm{CO}_{2}$ laser caused no statistically significant difference with the control group but clinically, the created surface roughness was considerable.

In a study by Shahabi et al in 2013 on extracted human molar teeth, different lasers were applied and it was reported that $1.5 \mathrm{~W}$ and $80 \mathrm{~Hz} \mathrm{CO}_{2}$ laser resulted in melting and crack formation on dentin surface. Evidence shows that $\mathrm{CO}_{2}$ laser with different parameters results in evaporation of water and dentin organic materials; therefore, surface roughness and melting of some dentin

No treatment
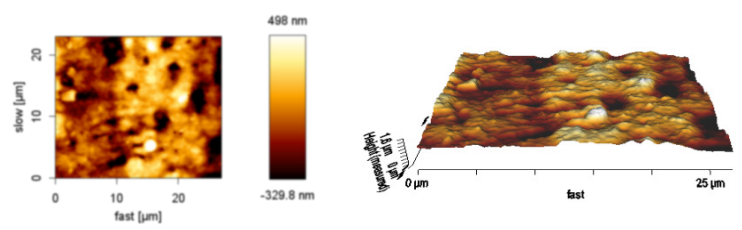

$\mathrm{Co}_{2}$
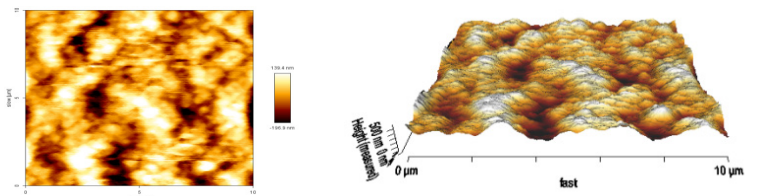

Er:YAG
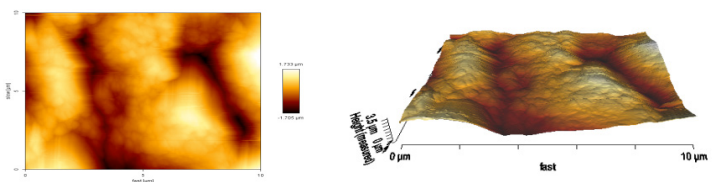

Figure 2. AFM Micrographs of the Diameter of Dentinal Tubules in the Samples. 
areas occur. In fact, cracks form due to tissue shrinkage following loss of water and collagen matrix. In general, use of standard laser parameters for effective hard tissue ablation is important for an expert operator. ${ }^{35}$

Rough surfaces result in formation and maturation of microbial plaque. Dental plaque causes periodontal disease and dental caries. This indicates the clinical importance of surface roughness. ${ }^{36}$

Some studies have evaluated diameter of dentinal tubules. ${ }^{19,20}$ Ehlers et al ${ }^{19}$ studied on 15 females and 7 males with cervical DH treated one quadrant with GLUMA, and the other quadrant with Er:YAG laser reported that laser therapy for treatment of $\mathrm{DH}$ was more effective than chemical agents for obstruction of dentinal tubules and decreasing their diameter.

$\mathrm{Yu}$ et al in 2012 showed that Er:YAG laser and $\mathrm{CO}_{2}$ laser were both effective for treatment of $\mathrm{DH}$ without adversely affecting the pulp. ${ }^{37}$

In 2010, Dilsiz et al, in Turkey evaluated the effects of Er:YAG (2940 nm, $60 \mathrm{~mJ}, 2 \mathrm{~Hz}, 20$ seconds), Nd:YAG (1064 nm, $100 \mathrm{~mJ}, 15 \mathrm{~Hz}, 100$ seconds) and diode laser (808 nm, $100 \mathrm{mw}, 20$ seconds) as dentin desensitizers in short and long term on teeth with gingival recession. They evaluated 24 patients and 96 teeth with gingival recession. The results showed significant reduction in $\mathrm{DH}$ at all measured time points in the 3 treatment sessions in groups treated with Er:YAG, Nd:YAG and diode lasers. ${ }^{38}$ Gholami et al in 2011 evaluated dentin surfaces exposed to $14 \%$ EDTA and showed that application of $1 \mathrm{~W} \mathrm{CO}$ laser melted the peri-tubular dentin and obstructed the dentinal tubules partially or completely. Thus, DH decreased. ${ }^{39}$

Using SEM, Gursoy et al, in 2012 indicated that Er:YAG laser irradiation $(30 \mathrm{~Hz}, 60 \mathrm{~mJ}, 10$ seconds) decreased the diameter and number of dentinal tubules. ${ }^{40}$

Evidence shows that laser irradiation of dentin surfaces results in surface destruction or melting of dentin hydroxyapatite crystals. Resultantly, dentinal tubules are obstructed and hydrodynamic stimulation of pulpal nerve fibers (which causes DH) no longer occurs. Moreover, laser irradiation changes the surface topography and dentin surface roughness. Increased plaque retention also compromises the periodontal health. Furthermore, our study indicated Laser irradiation of dentin surface affected the surface topography and surface roughness of dentin. Evaluation of changes in surface roughness parameters ( $\mathrm{Ra}$ and $\mathrm{Rq}$ ) after laser irradiation revealed that surface roughness increased following laser irradiation.

\section{Conclusion}

Based on the results of this study, Er:YAG laser irradiation can obstruct the dentinal tubules and probably decrease $\mathrm{DH}$. However, it can also increase surface roughness, microbial plaque retention, risk of periodontal disease, and dental caries. Surface roughness and obstruction of dentinal tubules occurred to a lesser extent by the application of $\mathrm{CO}_{2}$ laser (compared to Er:YAG); nevertheless, these effects were clinically considerable. Future studies are required on the same lasers with different irradiation parameters as well as on other types of lasers with dental applications to assess their efficacy for treatment of $\mathrm{DH}$.

\section{Conflict of Interests}

The authors declare no conflict of interest.

\section{Ethical Considerations}

Not applicable.

\section{References}

1. Holland GR, Narhi MN, Addy M, Gangarosa L, Orchardson R. Guidelines for the design and conduct of clinical trials on dentine hypersensitivity. J Clin Periodontol. 1997;24(11):808-813.

2. Meier ML, Brugger M, Ettlin DA, et al. Brain activation induced by dentine hypersensitivity pain--an fMRI study. J Clin Periodontol. 2012;39(5):441-447. doi:10.1111/j.1600051X.2012.01863.x

3. Graf H, Galasse R. Morbidity, Prevalence and Intraoral Distribution of Hypersensitive Teeth. J Dent. 1997;56:162165.

4. Bamise CT, Esan TA. Mechanisms and treatment approaches of dentine hypersensitivity: a literature review. Oral Health Prev Dent. 2011;9(4):353-367.

5. Bartold PM. Dentinal hypersensitivity: a review. Aust Dent J. 2006;51(3):212-218.

6. Gillam D, Orchardson R. Advances in the treatment of root dentine sensitivity: mechanisms and treatment principles. Endod Topics. 2006;13(1):13-33. doi:10.1111/j.16011546.2006.00209.x

7. Blaggana A, Vohra P, Nagpal A. Diagnosis and treatment of dentinal hypersensitivity. J Innov Dent. 2011;1(3):1-4.

8. Cohen S, Hargreaves KM. Pathways of the Pulp. In: Roberson T, Heymann HO, Swift EJ, eds. Sturdevant's Art and Science of Operative Dentistry. Elsevier Health Sciences; 2006.

9. Gernhardt CR. How valid and applicable are current diagnostic criteria and assessment methods for dentin hypersensitivity? An overview. Clin Oral Investig. 2013;17 Suppl 1:S31-40. doi:10.1007/s00784-012-0891-1

10. Collaert B, Fischer C. Dentine hypersensitivity: a review. Endod Dent Traumatol. 1991;7(4):145-152. doi:10.1111/j.1600-9657.1991.tb00200.x

11. Jeffcoat MK. Prevention of periodontal diseases in adults: strategies for the future. Prev Med. 1994;23(5):704-708. doi:10.1006/pmed.1994.1119

12. Matsumoto K, Funai H, Shirasuka T, Wakabayashi H. Effects of Nd:YAG laser in treatment of cervical hypersensitive dentin. Japan J Conserv Dent. 1985;28:760-765

13. Ranjan R, Yadwad K, Patil S, Mahantesha S, Rahman A, Bhatia V. Efficacy of $980 \mathrm{~nm}$ diode laser as an adjunct to $\mathrm{Snf}_{2}$ in the management of dentinal hypersensitivity: A controlled, prospective clinical study. J Dent Laser. 2013;7(2):66-71. doi:10.4103/0976-2868.124267 
14. Kimura Y, Wilder-Smith P, Yonaga K, Matsumoto K. Treatment of dentine hypersensitivity by lasers: a review. $J$ Clin Periodontol. 2000;27(10):715-721.

15. Moritz A, Gutknecht N, Schoop U, et al. The advantage of CO2-treated dental necks, in comparison with a standard method: results of an in vivo study. J Clin Laser Med Surg. 1996;14(1):27-32.

16. Moritz A, Gutknecht N, Schoop U, Wernisch J, Lampert F, Sperr W. Effects of $\mathrm{CO} 2$ laser irradiation on treatment of hypersensitive dental necks: results of an in Vivo study. $J$ Clin Laser Med Surg. 1995;13(6):397-400

17. Schwarz F, Arweiler N, Georg T, Reich E. Desensitizing effects of an Er:YAG laser on hypersensitive dentine. J Clin Periodontol. 2002;29(3):211-5.

18. Sgolastra F, Petrucci A, Gatto R, Monaco A. Effectiveness of laser in dentinal hypersensitivity treatment: a systematic review. J Endod. 2011;37(3):297-303. doi:10.1016/j. joen.2010.11.034

19. Ehlers V, Ernst CP, Reich M, Kammerer P, Willershausen B. Clinical comparison of gluma and Er:YAG laser treatment of cervically exposed hypersensitive dentin. Am J Dent. 2012;25(3):131-135.

20. He S, Wang Y, Li X, Hu D. Effectiveness of laser therapy and topical desensitising agents in treating dentine hypersensitivity: a systematic review. J Oral Rehabil. 2011;38(5):348-358. doi:10.1111/j.1365-2842.2010.02193.x

21. Meyer E, Hug HJ, Bennewitz R. Introduction to scanning probe microscopy. Scanning Probe Microscopy. Berlin Heidelberg; Springer: 2004:1-13.

22. Cury MS, Silva CB, Nogueira RD, Campos MGD, PalmaDibb RG, Geraldo-Martins VR. Surface roughness and bacterial adhesion on root dentin treated with diode laser and conventional desensitizing agents. Lasers Med Sci. 2018;33(2):257-262. doi:10.1007/s10103-017-2356-x

23. Nogueira RD, Silva CB, Lepri CP, Palma-Dibb RG, GeraldoMartins VR. Evaluation of Surface Roughness and Bacterial Adhesion on Tooth Enamel Irradiated With High Intensity Lasers. Braz Dent J. 2017;28(1):24-29. doi:10.1590/01036440201701190

24. Sgan-Cohen HD, Livny A, Vered Y. The elmex SENSITIVE toothbrush: effect on plaque reduction and subjective satisfaction after two months. J Clin Dent. 2008;19(1):2227.

25. Flecha OD, Azevedo CG, Matos FR, et al. Cyanoacrylate versus laser in the treatment of dentin hypersensitivity: a controlled, randomized, double-masked and noninferiority clinical trial. J Periodontol. 2013;84(3):287-294. doi:10.1902/jop.2012.120165

26. Goldberg F, Spielberg C. The effect of EDTAC and the variation of its working time analyzed with scanning electron microscopy. Oral Surg Oral Med Oral Pathol. 1982;53(1):74-77.

27. Yamada RS, Armas A, Goldman M, Lin PS. A scanning electron microscopic comparison of a high volume final flush with several irrigating solutions: Part 3. J Endod. 1983;9:137-42.

28. Pashley DH. Dentin permeability, dentin sensitivity, and treatment through tubule occlusion. J Endod. 1986;12(10):465-474. doi:10.1016/s0099-2399(86)80201-1

29. McCarthy D, Gillam DG, Parson DJ. In vitro effects of laser radiation on dentine surfaces. J Dent Res. 1997;76:233.

30. Crespi R, Barone A, Covani U. Er:YAG laser scaling of diseased root surfaces: a histologic study. J Periodontol. 2006;77(2):218-222. doi:10.1902/jop.2006.050043

31. Botta SB, Ana PA, de Sa Teixeira F, da Silveira Salvadori MC, Matos AB. Relationship between surface topography and energy density distribution of Er,Cr:YSGG beam on irradiated dentin: an atomic force microscopy study. Photomed Laser Surg. 2011;29(4):261-269. doi:10.1089/ pho.2010.2812

32. Schwarz F, Arweiler N, Georg T, Reich E. Desensitizing effects of an Er:YAG laser on hypersensitive dentine. J Clin Periodontol. 2002;29(3):211-215.

33. Birang R, Poursamimi J, Gutknecht N, Lampert F, Mir M. Comparative evaluation of the effects of Nd:YAG and Er:YAG laser in dentin hypersensitivity treatment. Lasers Med Sci. 2007;22(1):21-24. doi:10.1007/s10103-006-0412-z

34. Soares LES, Bitar RA, Brugnera A, et al. Er:YAG laser irradiation on dentin: FT-Raman and SEM studies. SPIE BiOS. 2007. doi:10.1117/12.702244

35. Shahabi S, Chiniforush N, Juybanpoor N. Morphological Changes of Human Dentin after Erbium-Doped Yttrium Aluminum Garnet (Er:YAG) and Carbon Dioxide (CO2) Laser Irradiation and Acid-etch Technique: An Scanning Electron Microscopic (SEM) Evaluation. J Lasers Med Sci. 2013;4(1):48-52.

36. Quirynen M, Bollen CM. The influence of surface roughness and surface-free energy on supra- and subgingival plaque formation in man. A review of the literature. J Clin Periodontol. 1995;22(1):1-14.

37. Yu C-H, Chang Y-C. Clinical efficacy of the Er:YAG laser treatment on hypersensitive dentin. Journal of the Formosan Medical Association. 2014;113(6):388-91.

38. Dilsiz A, Canakci V, Ozdemir A, Kaya Y. Clinical evaluation of Nd:YAG and 685-nm diode laser therapy for desensitization of teeth with gingival recession. Photomed Laser Surg. 2009;27(6):843-848. doi:10.1089/pho.2008.2395

39. Gholami GA, Fekrazad R, Esmaiel-Nejad A, Kalhori KA. An evaluation of the occluding effects of Er;Cr:YSGG, Nd:YAG, $\mathrm{CO}(2)$ and diode lasers on dentinal tubules: a scanning electron microscope in vitro study. Photomed Laser Surg. 2011;29(2):115-121. doi:10.1089/pho.2009.2628

40. Gursoy H, Cakar G, Ipci SD, Kuru B, Yilmaz S. In vitro evaluation of the effects of different treatment procedures on dentine tubules. Photomed Laser Surg. 2012;30(12):695698. doi:10.1089/pho.2012.3336 